\title{
ACCELERATORS DEVELOPMENT FOR INTRAOPERATIVE RADIATION THERAPY
}

\author{
C. Ronsivalle, L. Picardi, A. Vignati, ENEA-FIS, CR-Frascati, Italy \\ A. Tata, ENEA-FIS, CR-Casaccia, Anguillara (Roma), Italy, \\ M. Benassi, Istituti Fisioterapici Ospedalieri (IFO), Roma, Italy
}

\begin{abstract}
The "Intra Operative Radiation Therapy" (IORT) consists in delivering a single high dose on a tumour bed soon after surgery resection. The "IORT Project", funded by the Italian Ministry of Research (MURST), was started in 1998 from ENEA (Italian National Agency for New Technologies, Energy and the Environment) and is aimed to design and construct two innovative IORT systems (named IORT-1 and IORT-2) equipped with advanced treatment planning and real time dosimetry. These developments will improve the present IORTNOVAC7 system, jointly developed in the latest years from ENEA and the Italian Hitesys Company. In the paper the advance status of the project is described and IORT-1 experimental results are presented with particular attention to innovative technical aspects of these new generation IORT accelerators.
\end{abstract}

\section{INTRA-OPERATIVE RADIOTHERAPY}

The "Intra Operative Radiation Therapy" (IORT) [1] represents a very effective and emerging oncological treatment. The main advantage of IORT technique, in addition to the possibility to avoid additional radiotreatments after surgery intervention, is to be referred to the improved tumor local control [2]: as a matter of fact the technique allows the hitting with an high single dose focused only on diseased tissue, avoiding to irradiate healthy tissues and preventing tumor recurrence in surrounding area.

The IORT last generation equipment, that is represented by a very compact, light and mobile EB-machine located in the same operating room, overcomes, moreover, the previously foreseen patients transfer to the hospital radiotherapy shielded department just after the resection (patient's additional stress and anaesthesia, difficulties to preserve a sterile path during conveyance, etc.).

The first world equipment of such type was conceived by ENEA and the Italian Co. HITESYS, since early '90s, and named as NOVAC7 [3], and at present more than ten models of such equipment are actively operating by Italian and European hospitals with some hundreds successful treatments carried out.

The experience carried out on such system showed that, although it is one of the most advanced IORT equipments worldwide, some technical modifications can allow a meaningful improvement of present performances.
At this aim in 1998 ENEA launched the "IORT Project"[4], funded by the Italian Ministry of Research (MURST).

\section{NEW FRONTIERS FOR IORT}

\subsection{The ENEA-Hitesys NOVAC7 system}

The NOVAC7 system is a robotic mobile intraoperative electron beam unit (table 1).

It is based on a compact S-band standing wave electron beam linear accelerator utilizing a patented autofocusing structure which eliminates the need of focusing solenoids. It is characterized by small size, light weight and negligible diffused $\mathrm{X}$ radiation. The accelerator is moved by a six axis robotic arm. The dose is delivered to the patient's surgical breach, through a scattered electron beam collimated by plexiglas applicators, (the diameter varies from 40 to $120 \mathrm{~mm}$ ) attached to the radiating head.

Table 1: NOVAC7 technical data

\begin{tabular}{|c|c|}
\hline Surface dose & $\geq 80 / 85 \%$ \\
\hline Dose rate & $\geq 4$ and $\leq 31 \mathrm{~Gy} / \mathrm{min}$ \\
\hline Field size & $4,6,8,10 \mathrm{~cm}$ \\
\hline X-ray contamination & $\leq 0.3 \%$ \\
\hline Dimensions & $\mathrm{L}=2.3 \mathrm{~m}, \mathrm{~W}=1 \mathrm{~m}, \mathrm{H}=1.9 \mathrm{~m}$ \\
\hline
\end{tabular}

\subsection{IORT-1 system}

In the framework of the "IORT Project" a first prototype (IORT-1) was built and tested. In comparison with NOVAC7, from the point of view of the accelerator it is characterized by the possibility to modulate the output beam current, by an increased output beam energy and a wider energy variation range. These features were obtained using a different electron gun and a more efficient accelerating structure.

The need to change the NOVAC7 e-gun is due to the fact that as NOVAC7 does not employ scattering filters (that in the conventional machines are the main source of stray radiation), it is complicate to modulate accelerator dose rate, which is high comparing to conventional accelerators. This prevents the use of ionisation chambers that show a too high saturation factor due to the high dose rate. In the NOVAC7 the use of a diode gun does not allow to change the injected current without changing the injection voltage. In the IORT-1, in order to modulate the beam current preserving the beam characteristics at the linac input, a triode gun followed by an univoltage 
lens was designed. The electrodes geometry was optimised by using the EGUN code.

The second improvement of the IORT-1 system concerns the accelerating structure: the IORT-1 single cavities shape was optimized in order to maximise the efficiency and to reduce the dark currents, which could be a serious problem for the operation of the system at very low currents. The main characteristics of the two accelerating structures are compared in table 2 .

Table 2: NOVAC7 and IORT-1 accelerator parameters

\begin{tabular}{|l|c|c|}
\hline & NOVAC7 & IORT-1 \\
\hline Maximum energy & $9 \mathrm{MeV}$ & $11 \mathrm{MeV}$ \\
\hline $\begin{array}{l}\text { Typical energy } \\
\text { variation range (by } \\
\text { steps) }\end{array}$ & $4-8 \mathrm{MeV}$ & $3-10 \mathrm{MeV}$ \\
\hline Beam current & $1.5 \mathrm{~mA}$ & $0.1-1.0 \mathrm{~mA}$ \\
\hline Shunt impedance & $72 \mathrm{Mohm} / \mathrm{m}$ & $80.5 \mathrm{Mohm} / \mathrm{m}$ \\
\hline Linac Length & $50 \mathrm{~cm}$ & $70 \mathrm{~cm}$ \\
\hline $\begin{array}{l}\text { Number of cavities } \\
(\beta \text { graded) }\end{array}$ & 11 & 15 \\
\hline $\begin{array}{l}\text { Maximum } \\
\text { Kilpatrick Factor }\end{array}$ & 1.8 & 1.5 \\
\hline
\end{tabular}

PARMELA beam dynamics computations were done in order to check the matching between the new gun and the new structure and showed that the autofocusing feature is preserved.

In figure 1 the values beam current collected at the accelerator output for different values of the grid voltage are plotted and compared with the curve fitting the design values as computed by PARMELA dynamics calculations having assigned in input the distribution computed by EGUN.

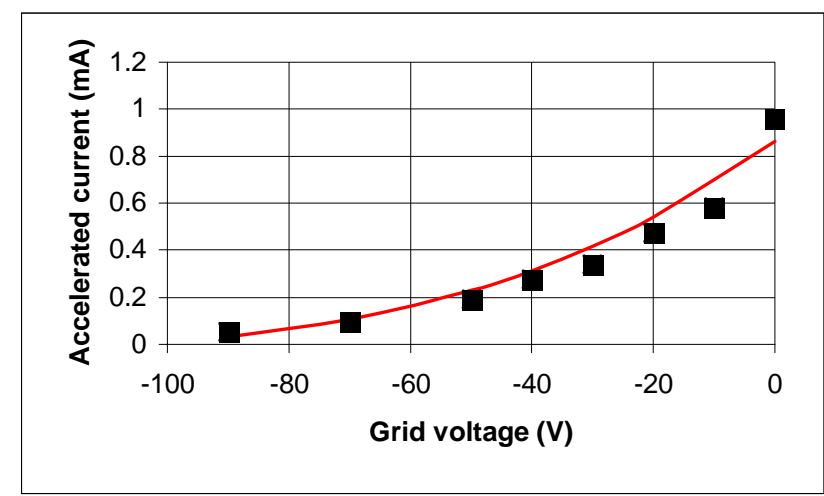

Figure 1: Measured variation of output beam current vs grid voltage: $\mathbf{m}$ measured data, _ computed curve

Depth dose measurements have been carried out in Scanditronix water phantom utilizing a ionisation chamber. From this type of measurement it is possible to derive the parameters that are relevant for irradiation: the average energy at the water phantom $E_{0}=2.33 R_{50}\left(R_{50}=\right.$ range corresponding to a dose equal to $50 \%$ of the maximum value also called HVD, half value depth in water), the so called most probable energy value $\mathrm{E}_{\mathrm{p} 0}=0.22+1.98 \mathrm{R}_{\mathrm{p}}+2.5 \cdot 10^{-3} \mathrm{R}_{\mathrm{p}}^{2} \quad\left(\mathrm{R}_{\mathrm{p}}=\right.$ practical range corresponding to the maximum range in water) and the range corresponding to a dose equal to the $80 \%$ of maximum dose $R_{80}$ (this is considered from radiotherapists the useful range of irradiation). We verified that the value of $\mathrm{E}_{\mathrm{p} 0}$ corresponds to the beam average energy as it is computed by dynamics calculations. The difference between the maximum beam energy and the average value is about $1 \mathrm{MeV}$.

Figures 2 and 3 compare NOVAC7 and IORT-1 system performances for four different values of magnetron input RF power $(1.1,1.4,1.7,2.4 \mathrm{MW})$ and a source surface distance $(\mathrm{SSD})$ of $100 \mathrm{~cm}$. The results are in good agreement with the design values.

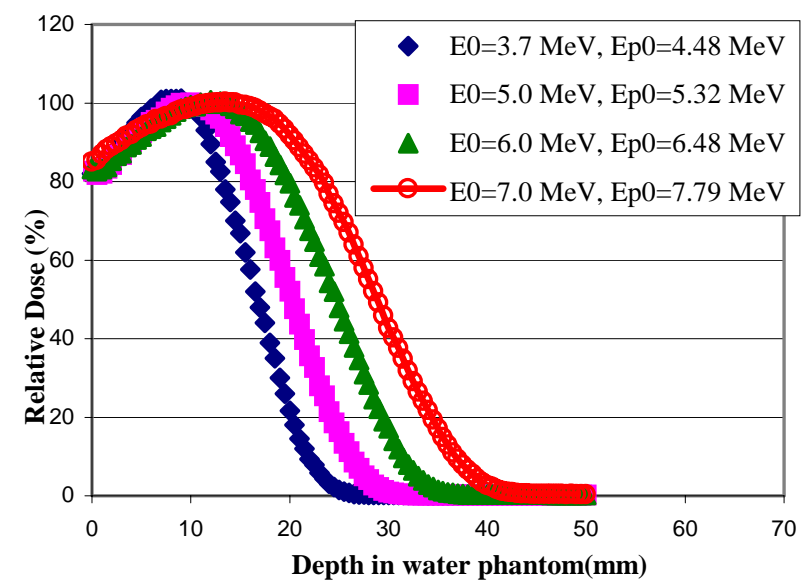

Figure 2: Measured dose penetration depth curves in water phantom for the NOVAC7 system

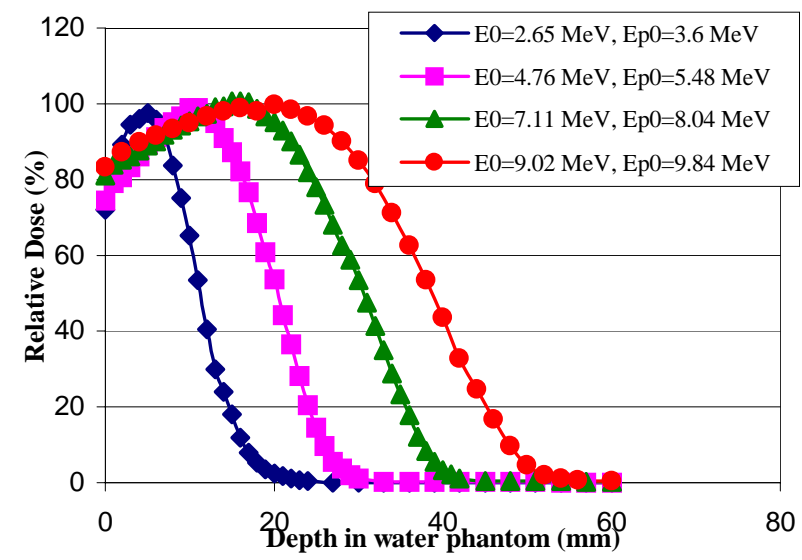

Figure 3: Measured dose penetration depth curves in water phantom for the IORT-1 system

Dark current measurements were done by the ionisation chamber. In the NOVAC7 at the maximum energy a dark current equal to $8 \%$ of the accelerated current is measured when the cathode is off and the accelerating structure is RF powered: it is due to electrons extracted from the surfaces by the high surface electric fields. No dark current was measured in the IORT1 at the maximum NOVAC7 energy and at IORT-1 maximum energy the dark current is less than $2 \%$. These results confirm the reduction of the surface electric field due to the new shapes of accelerating cavities. 


\section{TREATMENT PLANNING}

Knowledge of dose distribution that, in the specific situations relating to each patient, can be obtained using the I.O.R.T. irradiation technique is a decisive factor to optimize intra-operative radiation therapy. [5]

The distribution of the doses deposited by the beams of electrons can be known beforehand with sufficient reliability, through computer simulation of the real situations starting from significant data obtained directly from the patient or derived from previously acquired sequences of CT or NMR images and by simulating, with an adequate model, the electron beam and the relevant deposit of the dose on the volumes of [6] interest.

For the development of the treatment planning system, the following subjects have been studied and defined:

a) Detailed definition of the procedure for the acquisition of the patient three-dimensional anatomical data and identification of the best course of operation and the best technique of immobilization, recognition and target hitting, through anatomical and other findings, using available diagnostic instruments: CT, Simulation and MR.

The number of CT images needed to describe adequately the irradiated volumes and the organs to spare has also been defined; additionally, a software module has been devised for the transmission of images from the acquisition units to the computer of the system to simulate the treatment.

b) Detailed definition of the necessary information for an accurate outline of the PTV (Planning Target Volume) and the critical organs.

For the processing and graphic visualization of the patient's clinica-dosimetric data, a dedicated software modules have been identified and tested. As part of the graphic display of the patient's data, an algorithm has been implemented for processing and 3D display of the patient's CT data and for the $3 \mathrm{D}$ reconstruction of VOI (volume of interest) starting from their 2D contours. As far as the segmentation of ROI (regions of interest) is concerned, some routines for manual contouring have been devised, in graphic environments.

c) Detailed definition of the algorithms and software for the calculation of the dose which is written basically in the FORTRAN language.

A first software module has been implemented to display the output of the calculation codes, in other words how the dose is distributed within the patient body, in the form of isodose curves in transparency over the CT images, and in 3D presentation, as isodose surfaces, superimposed onto the patient's 3D reconstruction (fig. 4).

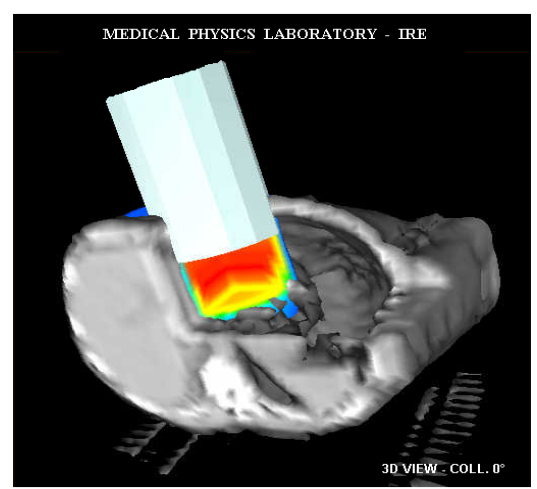

Figure 4: A three-dimensional virtual reconstruction of the patient with an IORT collimator inserted, having a diameter of $10 \mathrm{~cm}$ and a $0^{\circ}$ angle of exit of the beam. The dose distribution of the electrons of 9 $\mathrm{MeV}$ is shown in the trasparency.

\section{NEXT DEVELOPMENTS}

The "IORT Project" foresees the construction of a second system named IORT-2 provided with a more powerful magnetron (3.1 MW instead of 2.6 MW), utilizing the IORT-1 radiating head, and able to release the dose by beam mechanical scanning on the surgical field. This kind of irradiation called "incremental irradiation" is particularly suitable for the distribution of conformational doses on fields of irregular shape.

In order to enable such developments, some small modifications are required: the robotic arm should be able to perform planned movement over complex surfaces, a proper simulation of this new radiation treatment must be developed and appropriate real-time dosimetric systems set up. Studies regarding above referred points are in advanced progress.

\section{REFERENCES}

[1] Proc. of the $6^{\text {th }}$ International IORT Symposium and $31^{\text {st }}$ San Francisco Cancer Symposium, San Francisco (U.S.A.), 22-25 sept. 1996.

[2] Proc. of the ISIORT ' 98 Conf.- $\mathrm{I}^{\text {st }}$ Congress of the Intern. Soc. of Intraoperative Therapy, University of Navarra, Pamplona (Spain), 6-9 sept. 1998.

[3] M.Fantini, et al., "IORT Novac7: A New Linear Accelerator for Electron Beam Therapy", in "Intraoperative Radiation Therapy in the Treatment of Cancer", Front. Radiat. Ther. Oncol., Vaeth J.M. editor, Basel, Karger, 1997, vol. 31, pp.54-59.

[4] L. Picardi, C. Ronsivalle, A. Tata, A. Vignati "The Italian IORT Project”, Proc. EPAC2000, Vienna, p. 2545

[5] M: Rosetti, M: Benassi, V. Bruzzaniti, A. Bufacchi, M. D'Andrea “ Intra-operative Radiation Therapy Optimization Usimg the Monte Carlo”, Proc. Monte Carlo 2000, Lisbon.

[6] M: Benassi, L. Begnozzi, A, Bufacchi, R. Fragomeni, A. Tata,. Creton "Treatment Plans: Some Help for IORT”, Proc. SIRR-INFN-ENEA, Padova 2000. 